\title{
Sleep, recovery, and metaregulation: explaining the benefits of sleep
}

\author{
This article was published in the following Dove Press journal: \\ Nature and Science of Sleep \\ 17 December 2015 \\ Number of times this article has been viewed
}

\section{Vladyslav V Vyazovskiy}

Department of Physiology, Anatomy, and Genetics, University of Oxford, Oxford, UK
Correspondence: Vladyslav $\vee$ Vyazovskiy Department of Physiology, Anatomy, and Genetics, University of Oxford, Parks Road, Oxford OXI 3PT, UK Email vladyslav.vyazovskiy@dpag.ox.ac.uk
Abstract: A commonly held view is that extended wakefulness is causal for a broad spectrum of deleterious effects at molecular, cellular, network, physiological, psychological, and behavioral levels. Consequently, it is often presumed that sleep plays an active role in providing renormalization of the changes incurred during preceding waking. Not surprisingly, unequivocal empirical evidence supporting such a simple bi-directional interaction between waking and sleep is often limited or controversial. One difficulty is that, invariably, a constellation of many intricately interrelated factors, including the time of day, specific activities or behaviors during preceding waking, metabolic status and stress are present at the time of measurement, shaping the overall effect observed. In addition to this, although insufficient or disrupted sleep is thought to prevent efficient recovery of specific physiological variables, it is also often difficult to attribute specific changes to the lack of sleep proper. Furthermore, sleep is a complex phenomenon characterized by a multitude of processes, whose unique and distinct contributions to the purported functions of sleep are difficult to determine, because they are interrelated. Intensive research effort over the last decades has greatly progressed current understanding of the cellular and physiological processes underlying the regulation of vigilance states. Notably, it also highlighted the infinite complexity within both waking and sleep, and revealed a number of fundamental conceptual and technical obstacles that need to be overcome in order to fully understand these processes. A promising approach could be to view sleep not as an entity, which has specific function(s) and is subject to direct regulation, but as a manifestation of the process of metaregulation, which enables efficient moment-to-moment integration between internal and external factors, preceding history and current homeostatic needs.

Keywords: sleep, wakefulness, sleep homeostasis, sleep regulation, exercise, EEG

\section{Introduction}

Sleep is a universal behavior occupying a significant fraction of the 24-hour day, ${ }^{1,2}$ but its regulation and function(s) are far from being understood. ${ }^{3-5}$ There is an extensive amount of data and many theories, which suggest that sleep plays an active role in processes such as synaptic plasticity and memory functions, ${ }^{6-8}$ emotional regulation, ${ }^{9-11}$ metabolic functions and energy balance, ${ }^{12-17}$ macromolecule biosynthesis, ${ }^{18}$ removal of toxic substances and metabolic waste, ${ }^{19,20}$ or prophylactic cellular maintenance. ${ }^{5}$ Currently, none of these theories are generally accepted and some of them show considerable overlap. More recently, there has been a shift toward a novel view, which postulates that the beneficial role of sleep is related to it being a default state of the organism or cerebral networks ${ }^{21-24}$ or a state of adaptive inactivity. ${ }^{25}$ This view helps to explain the wide variability in the amount and architecture of 
sleep between species, ${ }^{26}$ which can be accounted for by specific ecological factors including a difference in time needed to fulfill waking activities, such as feeding or reproduction.

One widely held view is that the need for sleep increases as a function of time spent awake, and that sleep is necessary to provide restoration with respect to molecular, cellular, or network changes, which occur specifically during preceding waking. ${ }^{27,28}$ This view is based on the notion that sleep is a regulated process, where several factors such as time of day, preceding waking duration, or specific types of activity are important determinants of the duration and intensity of sleep. ${ }^{29} \mathrm{~A}$ key observation is that the effects of various factors, acting at a specific moment of time (eg, ambient temperature) and reflecting preceding history, are revealed during specific changes in sleep and can be interpreted in terms of their involvement in sleep regulation. However, an alternative interpretation is that the essential characteristics of sleep, such as specific brain oscillations and the timing or duration of sleep, merely reflect other processes taking place at the time of measurement. The theoretical concept of metaplasticity is useful in aiding this discussion. Metaplasticity is considered as a higher-order form of plasticity and refers to the process that regulates the induction of plastic changes. ${ }^{30}$ For example, preceding history of activity, the levels of neuromodulators, ongoing synaptic state, or the global synaptic weight of the entire network are powerful modulators of the magnitude and direction of plasticity occurring at the level of individual synapses. In analogy with this concept, rather than considering sleep as a primary target of regulatory processes, it can be viewed as a process of metaregulation, that is a higher-order form of regulation, which accommodates a broad range of molecular, cellular and network processes altogether providing optimal (adaptive) wakefulness.

We define adaptive waking as a condition whereby the organism interacts effectively with the environment and is capable of maintaining normal physiological waking functions within strict physiological limits, which altogether increases chances of survival and successful reproduction. It should be pointed out that the terms "waking" and "sleep" did not originally have a scientific connotation, but were rather concepts defined qualitatively (for example, when referring to the absence of movement or a body posture), or based on the analysis of subjective experience. Nevertheless, this terminology persists today and is widely used in scientific literature, despite the terms often being too restrictive or inadequate for specific cases. It is worth noting that wakefulness, and in particular adaptive waking (as defined above), is a highly heterogeneous phenomenon. In simplistic terms, different types of waking can be classified based on the amount and character of movement or specific kinds of behavior. It is germane for this discussion, therefore, to introduce further subdivisions of waking, specifically into "low cost" and "high cost" elements, both with respect to energy and resources required for specific behaviors, and to their effects on future states. On the other hand, it is important to keep in mind that "sleep" is also an infinitely complex phenomenon, characterized by many interrelated processes. ${ }^{28}$ Some of these are relatively sleep specific, such as sleep oscillations ${ }^{31}$ or hormonal changes, ${ }^{32}$ while others are rather secondary processes and reflect the absence of sensory input and active locomotion, or physiological changes typical to a specific circadian time. As is the case for many other biological processes, the amount and dynamics of both waking and sleep are flexible, which is manifested on one hand as a considerable interindividual and interspecies variability, ${ }^{33-37}$ and on the other hand, in the capacity to stay awake beyond habitual sleep time, which can be modulated by involvement in specific behaviors, feeding and environmental changes such as ambient temperature and light.

The central theme to this article is that given a complex interdependency between physiological, behavioral, and psychological variables, as well as the unique effects of preceding and current state, it is difficult to estimate the pure effect of sleep or a lack thereof on any specific variable. For example, at the molecular level, mistimed sleep or sleep deprivation (SD) is associated with an altered transcription or translation of a wide range of molecules, which are implicated in core circadian rhythms generation both centrally ${ }^{38,39}$ and in the periphery, ${ }^{40}$ and are also involved in a wide range of other fundamental physiological processes. ${ }^{41}$ At the neuroanatomical level, circuits involved in energy homeostasis, waking, and sleep also show a considerable overlap, suggesting intimate links between them. ${ }^{42-45}$ At the behavioral level, effects of SD or preceding sleep on cognitive functions or psychomotor vigilance may be influenced by motivation or state instability.

In this article, first, the defining characteristics of sleep will be briefly considered. Then, some of the existing evidence for a role of sleep in recovery processes will be discussed, focusing on specific sleep-related phenomena such as the homeostatic regulation of sleep, the response to physical activities and the beneficial effects of sleep on subjective and objective measures of waking and performance. Finally, the concepts of recovery sleep and sleep regulation will be critically discussed. 


\section{What is it like to be asleep?}

Sleep is traditionally defined as a behavioral state that encompasses several essential characteristics used as defining markers, such as immobility or reduced behavioral responsiveness to external stimuli. ${ }^{3,33,46}$ Although these features are common across humans and animal species, a critical analysis reveals that they are often neither sufficient nor necessary for the classification of sleep, and their relevance for sleep regulation is not clear.

With regard to the absence of locomotor activity and voluntary movement, the question arises whether it is a necessary prerequisite or a consequence of a sleeping state, or both. One possibility is that immobility is necessary to prevent the flow of sensory information from proprioceptors, such as Golgi tendon organ or muscle spindles, to higher-order brain regions, which could otherwise interfere with ongoing spontaneous sleep activity. This is supported by recent evidence, which suggests that movement is associated with membrane depolarization in the neocortex ${ }^{47,48}$ while stimulation of the motor cortex during anesthesia affects sleep-like patterns of activity even in distant cortical regions. ${ }^{49}$ Alternatively, it is possible that cerebral activity during sleep, characterized by regular cessations of spiking and synaptic activity across widely-distributed brain areas, effectively disrupts large-scale network processing which is necessary for initiating and generating complex voluntary movements. Indeed, most of the brain is known to be involved to some extent in aspects of movement such as sensorimotor coordination, movement planning, and execution, which entail offline emulation of the body and the environment, ${ }^{50}$ required for efficient solving of various kinematic and dynamic tasks. ${ }^{51}$ Yet another possibility is that reduced locomotion during sleep is merely a consequence of reduced activity of those subcortical neuromodulatory areas, which are implicated both in maintaining arousal or other waking functions and necessary for movement, but can be, at least in part, dissociated from the sleeping state itself. In summary, while the absence of movement correlates with the occurrence of sleep, its relevance remains uncertain and further criteria are necessary for defining sleep.

Along the same line, disconnection from the sensory world may also be both a cause and a consequence of the sleeping state. An early study suggested that individual neurons may transition into "sleep-like" activity, despite the animal continuing to respond to stimuli. ${ }^{52}$ More recently, it was found that cortical neurons in the auditory cortex maintain responsiveness to auditory stimuli of various characteristics and modality while the animal remains asleep, unless the stimulus intensity is too high, when a startle response can trigger an arousal. ${ }^{53}$ This study led the authors to hypothesize that while primary sensory areas may be still responsive, the propagation of the stimulus to higher-order brain areas required for a global awakening is prevented by ongoing neuronal activity. This hypothesis is supported by the evidence that electrical microstimulation of a local cortical area during spontaneous non-rapid eye movement (NREM) sleep in rats elicits a stronger or attenuated response depending on background activity in the target area. ${ }^{54}$ Subcortical neuromodulatory areas proposed to be essential for global state transitions and information processing ${ }^{55}$ have also been shown to have increased activity close to an arousal. ${ }^{56-58}$ However, hypocretin neurons also may increase firing in response to auditory stimuli even when the animal remains asleep. ${ }^{56}$ This therefore suggests that both primary sensory cortical areas and subcortical regions are involved in monitoring the environment, but other factors are essential for triggering a global behavioral arousal. Moreover, optogenetic stimulation of hypocretin neurons did not necessarily lead to an immediate awakening, but rather increased the probability of an arousal in the next minute and prolonged the latency to sleep, especially during deep sleep. ${ }^{59}$ Thus, although sensory disconnection is an essential characteristic of sleep, it appears that the sleeping brain remains active in processing sensory information, and may even have capacity to extract task-related information, which indicates the presence of covert higher-order cognitive processing. ${ }^{60}$

As is well known, there is a considerable overlap between neurotransmitter systems involved in maintaining behavioral arousal, sensory responsiveness, and locomotion. ${ }^{1,61-64}$ These are used as defining characteristics for wakefulness, but are also crucial for such higher-order waking functions as neurobehavioral performance, attention, and cognition, which are affected to some extent by prolonged waking or by the time of day. ${ }^{65-67}$ An essential process in the initiation of sleep is a decreased activity in subcortical "wakepromoting" nuclei, which leads to characteristic changes in the electroencephalogram (EEG) and neuronal and activity in the forebrain. ${ }^{68,69}$ The occurrence of EEG slow waves is an essential defining characteristic of physiological NREM sleep, the polysomnogram detection of which is dictated by the amplitude and frequency of these slow waves, in addition to other electrographic phenomena such as spindles. However, as has been acknowledged by many, slow waves and their underlying cellular and network counterpart - the slow oscillation - can be dissociated from physiological NREM sleep. ${ }^{70,71}$ For example, sleep-like activity has been recorded in brain slices in vitro, ${ }^{72}$ under anesthesia, ${ }^{73,74}$ in isolated 
cortical slabs, ${ }^{75}$ during local application of norepinephrine antagonists, ${ }^{76}$ and during quiet waking. ${ }^{47,48}$ Moreover, slow waves during sleep can be reliably induced by electrical, auditory, or transcranial magnetic stimulation or by sensory inputs from periphery. ${ }^{54,77-80} \mathrm{An}$ interesting observation is that sleep has features of a "default state", such that neuronal cultures or isolated cortical slabs spontaneously generate patterns of activity similar in some respects to cortical activity during physiological sleep..$^{21,22,81}$ Notably, although it is sometimes questioned whether cortical EEG slow-wave activity (SWA, EEG power below $4 \mathrm{~Hz}$ ) is essential for sleep or sleep regulation, it remains a key measure for defining sleep in experiments where subcortical systems are targeted. ${ }^{82}$

The lack of progress in establishing direct links between states of vigilance, overt behaviors, and specific EEG activities has been acknowledged for some time.$^{83,84}$ Important insights can be obtained from focusing on specific phenomena characterizing waking and sleep such as their architecture, spatio-temporal dynamics of brain activity, ${ }^{85,86}$ as well as their regulation. ${ }^{87-89}$ More recently, it has been proposed that homeostatic regulation of sleep is its essential feature. ${ }^{26}$ It can be argued that while EEG slow waves per se may merely reflect a specific network state, ${ }^{24}$ the amount of excitatory input, ${ }^{81}$ and lowered levels of arousal-promoting neuromodulators typical for quiet wakefulness and immobility, ${ }^{47,48,90}$ their homeostatic regulation suggests an additional functional aspect, which allows to distinguish sleep from other conditions.

\section{Homeostatic regulation of sleep and EEG SWA}

Homeostatic regulation of sleep refers to the capacity to sleep longer or more intensely to compensate for sleep deficit. According to its original formulation, the homeostatic principle can be summarized as "the longer is the period of waking, the deeper or longer is subsequent sleep". ${ }^{88,89}$ $\mathrm{SD}$ is one of the main tools used to assess the integrity of homeostatic sleep regulation mechanisms in both animals and humans. ${ }^{91}$ Early studies utilizing SD or chronic sleep curtailment protocols reported consistent effects of SD on specific sleep stages. In one early study, young male subjects, who slept only 3 hours per day for eight consecutive days showed increased stage 4 sleep during sleep opportunity periods, ${ }^{92}$ and these results were corroborated by another follow-up human study. ${ }^{93}$ Likewise, in dogs, 12-hour forced wakefulness led to an increase in delta wave sleep during the first half of the night. ${ }^{94}$ In a quantitative EEG study, delta band power density was found to be significantly higher after SD compared with baseline for total sleep time as well as for sleep stages 2,3 , and $4 .^{95}$ Notably, and consistent with many follow-up studies, the effects observed were not restricted to the traditional delta band, but also extended into higher frequencies. Importantly, while the levels of SWA are invariably higher after SD, ${ }^{96}$ it has been shown that even relatively short periods of spontaneous waking lead to a predictable progressive increase in subsequent SWA, ${ }^{97,98}$ which led to the notion that it is a sensitive measure of sleep intensity. Interestingly, the amount of sleep or the levels of SWA before 6-hour SD affect the initial recovery sleep after $\mathrm{SD},{ }^{99}$ suggesting that sleep history leaves a trace, which is long lasting and resistant to erasure.

Notably, apart from the well-known overall decline of spectral power in the slow-wave frequency range with decreasing sleep pressure, there is also a redistribution of power toward lower frequencies, resulting in a shift in the spectral peak. As a consequence, several studies have reported a dissociation between the time course of EEG power in frequencies $<1 \mathrm{~Hz}$ and the remaining frequencies within the slow-wave range. ${ }^{100-103}$ Computer simulations revealed that a decrease in slow-wave incidence could fully account for an overall decline of SWA. ${ }^{104}$ At the same time, the dominant oscillatory activity manifested as a peak on the spectrum at a specific frequency is affected by the shape of slow waves, as is suggested by studies employing periodamplitude analysis that showed an increase in slow-wave period with decreasing sleep pressure. ${ }^{105-107}$ In simulations where slow-wave amplitude and incidence were kept constant, but the slope of slow waves was varied, a shift of the spectral power was observed, supporting the in vivo data. ${ }^{104}$ Consistently, in both animals and humans a decrease in the slope of slow waves was found with decreasing homeostatic sleep pressure. ${ }^{104,108,109}$ Thus, while decreased incidence of high amplitude slow waves may account for the overall lower SWA values, a reduction in slow-wave slopes may lead to the occurrence of spectral peak corresponding to slower frequencies. Such changes may arise at the level of cortical neuronal circuits, as it has been shown that early intense sleep encompassing large and frequent slow waves appeared to be associated with short, intense neuronal ON periods (periods of elevated neuronal activity), alternating frequently with relatively long OFF periods (periods of generalized population silence). ${ }^{110,111}$

Surprisingly, while homeostatic regulation of sleep is a precise, ubiquitous and basic phenomenon found in all animal species studied up-to-date, ${ }^{3,112}$ its underlying mechanisms are still unknown. There are several candidate mechanisms that are believed to be implicated in sleep 
need, including the regulation of brain metabolism, ${ }^{113-115}$ activity-dependent release of cytokines, ${ }^{116,117}$ and synaptic plasticity. ${ }^{118,119}$ The compensatory increase in SWA after a period of waking in proportion to its duration and the decline of SWA after sleep have been considered correlates of a "recovery" process occurring during sleep. The proposed changes requiring renormalization are diverse and span from energy homeostasis ${ }^{12,16}$ and removal of toxic products of metabolism ${ }^{20}$ to membrane repair and macromolecules biosynthesis. ${ }^{18,22,120-122}$ However, whether these recovery processes are actually taking place and what exactly is being recovered during sleep is still a matter of debate and will be considered next.

\section{Sleep need, sleep debt, and recovery sleep}

Sleep need, sleep debt, and recovery sleep are widely used concepts, in both scientific literature and beyond. Searches on these and related phrases return many hundreds citations in PubMed and countless web pages. "Recovery" is usually defined as regaining something that has been lost or taken away, or to restore or return to any former (better) state or condition. It is often concluded or implied that at least one of the functions of sleep is to provide recovery, and that the need to sleep or sleep debt reflects the need to obtain sufficient recovery. In addition, recovery is mentioned frequently when referring to a "compensatory" increase (rebound) in sleep time or sleep intensity after SD. Surprisingly, the literature demonstrating unequivocal evidence for recovery which can be attributed exclusively to sleep is scarce if not non-existent - the aspect that is rarely considered critically. Consistently, the role of sleep with respect to a specific variable is often inferred from the changes observed in the absence of sleep. However, the effects seen in conditions of insufficient sleep may rather reflect regulatory processes or a manifestation of a drive to initiate sleep rather than consequences of the lack of sleep itself. Arguably, SD is a replacement of sleep with other behaviors or states, rather than merely its absence. For example, if specific circuits requiring "recovery" enter sleep-like states after a certain amount of activity, ${ }^{5}$ the altered network dynamics would likely affect a variety of centrally-regulated functions, which would manifest itself at the level of behavior or metabolic disturbances. However, it does not necessarily mean that sleep normally plays a role in restoring those changes.

On a more fundamental level, in order to rigorously assess the recovery role of sleep it is necessary to compare pre-sleep with post-sleep values, a process that involves multiple confounding factors that are difficult to disentangle from one another. Specifically, the initial pre-sleep value of the variable under scrutiny does not only reflect the current state at the moment of measurement, but is also determined by the combined effects of preceding waking duration and specific waking activities, which are in addition influenced by the circadian phase. ${ }^{123}$ Subsequent sleep is in turn affected by preceding waking duration and specific activities, ${ }^{124}$ as well as by direct circadian effects on sleep, including its overall structure, duration, specific sub-stages, and brain oscillatory activities. ${ }^{125,126}$ Factors such as light ${ }^{127}$ and ambient temperature are often also crucial. ${ }^{128}$ As a result, any postsleep measurement represents a combined effect of all the factors mentioned above, including preceding long-term sleep-wake history and time of day, on the variable itself and on the physiological state during measurement, as well as the effects of immediate preceding state.$^{85,86,129} \mathrm{As}$ it is difficult to disentangle all of the aforementioned and control for each, this may explain discrepant findings, for example, with respect to effects of waking and sleep on synaptic strength or cortical firing rates. ${ }^{11,130-133}$

\section{Sleep and recovery after physical fatigue}

Since one of the main defining characteristics of sleep is the absence of movement, the question arises how and whether physical activity affects subsequent sleep. This question is also important since the compensatory rebound of sleep or sleep intensity after SD may depend on specific types of behavior, cognitive activities, or merely locomotor activity, which is often elevated when the animals or humans are sleep-deprived. Furthermore, it can be expected that sleep is beneficial for reversing tiredness typical after exercise. For these reasons, the role of sleep in recovery has been studied extensively in relation to exercise, both in animals and in humans. ${ }^{134}$

Early studies suggested that sleep facilitates recovery of the fatigue acquired during exercise. In one of the first experiments, cats that were subjected to treadmill running were found to have compensatory sleep changes that manifested as a decrease in sleep latency and an increase in synchronized EEG activity. ${ }^{135}$ These data were supported by the observation that rats showed an increase in daily sleep time after SD by forced treadmill activity. ${ }^{136}$ The hypothesis that sleep is associated with physical fatigue was also tested in a study where several different regimens of exercise were studied in two human subjects. ${ }^{137}$ This study found a whole-night increase in slow-wave sleep (SWS) that was related to the amount of physical fatigue, while the amount of rapid eye movement (REM) sleep was reduced. These 
results have since been confirmed in a more recent study, ${ }^{138}$ while another study found a consistent increase in SWA and stage 2 sleep, accompanied by a reduction in REM sleep in fit subjects across four conditions varying by the amount of exercise (running). ${ }^{139}$ However, a follow-up study from a different laboratory, in which eight subjects underwent either morning or evening exercise identified contradicting results. ${ }^{140}$ Specifically, while evening exercise resulted in an increase in stage 3 during subsequent sleep, morning training did not lead to any changes in sleep, which led the authors to conclude that recovery from muscle fatigue does not require sleep, and can instead be fulfilled during waking.

In rats, SD by forced locomotion on a "water wheel" resulted in an increased amplitude of the EEG recordings and intrusion of microsleeps during waking, while the subsequent sleep showed an increased level of high amplitude slow waves during NREM sleep. ${ }^{141}$ It is important to mention that it is unclear whether locomotor activity or waking duration per se accounts for this effect. In a control group, which had been awake for the same duration but walked much less, the rebound of sleep was similar, leading the authors to suggest that exercise per se has little influence on sleep. This notion was supported by a study showing that the effects of 12-hour SD by forced locomotion in rats were similar irrespective of wheel turning speed. ${ }^{142}$ It should be noted, however, that forced exercise may have different effects from voluntary exercise. ${ }^{143}$ In cats, it was found that more physical exercise was associated with a larger increase in SWS during subsequent sleep. ${ }^{144}$ It cannot be excluded that differences may be due to other factors such as the cognitive and motor effort needed to keep up with the rotation of the treadmill or even attempts to counteract the treadmill motion and accompanying stress associated with this. A similar conclusion has been reached in a human study where six men would march for $34 \mathrm{~km}$ each day with 5 hours rest allowed between the end of exercise and the subsequent sleep opportunity onset. ${ }^{145}$ The effects on subsequent sleep were variable between individuals however seemed to correlate with the levels of urinary 17-hydroxycorticosteroids. In another study, cycling for 3 hours also did not affect subsequent sleep. ${ }^{146}$ An interaction between exercise and SD was recently documented in a study where the exercise-related increase in growth hormone was elevated in sleep-deprived individuals. ${ }^{147}$

A significant limitation of human studies is that obvious technical difficulties prevent artifact-free EEG recordings during running per se. It was suggested that exercise is closely linked to cognition and does not entail merely physical effort, and this may underlie beneficial effects of exercise on cognition in aging. ${ }^{134}$ Interestingly, in overweight adults, exercise led to a reduction in default mode network activity in the precuneus. ${ }^{148}$ Another recent study found that functional connectivity in the default mode network was increased following 6-12 months of training, which led the authors to suggest that it reflects experience-dependent plasticity, as it was associated with an improvement in executive function. ${ }^{149}$

Notably, exercise appears to affect specific aspects of the compensatory sleep response. Specifically, in a rat study it was found that both EEG wave incidence and amplitude are responsive to prior wakefulness, but only incidence was influenced by prior exercise. ${ }^{107}$ This result is interesting given the different changes in network activity associated with specific parameters of EEG slow waves. Increased slow-wave incidence may suggest higher excitability or bistability of cortical networks, rather than increased network synchrony, which is associated with higher slow-wave amplitude as a result of longer and more profound population silence $\mathrm{e}^{111,150}$

Rodent studies have also shown that access to running wheels can substantially affect the distribution, amount, and architecture of vigilance states and activity across 24 hours, ${ }^{151,152}$ as well as EEG SWA. ${ }^{98}$ Notably, frontal predominance of SWA was enhanced after a period of waking without running wheel activity leading the authors to hypothesize that more diverse waking behaviors and activity led to a higher need for local or global "recovery". ${ }^{88}$ An intriguing observation was that when access to the wheel was not prevented, the duration of the first spontaneous waking period after dark onset was substantially extended compared with the wheel block condition. ${ }^{98}$ While this may suggest that in the absence of engaging activity the mice withdraw from active behaviors and therefore fall asleep earlier, it may also suggest that exploratory or diverse behaviors lead to a faster accumulation of sleep need as compared to stereotypical running behavior.

In summary, it is not surprising that variable and discrepant results have been obtained in studies addressing the effects of exercise on sleep, or assessing the "recovery" role of sleep after physical fatigue. It is likely that a simple relationship cannot be expected, as the effects of exercise may be strongly determined by the kind of exercise. This example highlights one of the difficulties in investigating global recovery functions of sleep. The next chapter will discuss briefly the role of sleep in central local mechanisms underlying behavioral performance.

\section{Does sleep recover behavioral performance and cognitive function?}

It has been argued that prolonged wakefulness leads to a progressive impairment of fundamental waking functions 
such as attention, learning, sensorimotor integration, and a range of executive cognitive functions. ${ }^{65,153,154}$ The mechanisms underlying the deleterious effects of insufficient or disrupted sleep are unclear and may be related to the lack of sleep (cumulative effects of preceding history) or merely reflect the tendency to enter sleep during the task (state dependency). By targeting relevant brain circuits in a selective way it is possible to investigate whether insufficient or disrupted sleep affects specific behaviors. Alternatively, cognitive and behavioral deficits may be secondary to a more fundamental global breakdown occurring at molecular, cellular, or network levels, which are incompatible with wakefulness in general. ${ }^{155}$

There is extensive evidence for use or activity dependency of sleep at the level of specific neural circuits. ${ }^{156}$ These studies were triggered by an observation that during spontaneous sleep SWA is not uniform across the cortical surface, but shows topographic gradients. In both humans and animals, SWA is more intense in the frontal derivations, especially during early sleep or after SD. ${ }^{157-162}$ Such changes may arise from anatomical differences, but could also be related to differential activation of specific brain regions. Specifically, studies have shown that peripheral stimulation or spontaneous use of circumscribed cortical areas leads to more intense EEG slow waves localized to the corresponding cortical regions. ${ }^{163-165}$ In addition, local, topographically distinct enhancement of slow waves was associated with learning of a motor task, ${ }^{124,166,167}$ while unilateral arm-immobilization led to a local decrease in slow waves. ${ }^{168}$ While a substantial body of evidence has been accumulated showing local activitydependent changes in sleep SWA, it is important to keep in mind that the effects are usually modest, rarely exceeding $10 \%-20 \%$, and by far lower than global wake-dependent changes. Nevertheless, such observations suggest that waking activities could affect the intensity of subsequent sleep in a selective manner, and that local changes in sleep activities may provide localized restoration. Activity-dependent sleep theories have implied that specific waking activities may lead to a selective disruption at the level of specific brain circuits resulting in their deterioration. ${ }^{67,169}$ Unfortunately, while many studies report deleterious effects of SD on specific neurobehavioral and cognitive functions, relatively few specifically addressed whether sleep can reverse the observed changes, and whether the effects are truly sleep specific.

An early study used visual stimulation during waking to test the hypothesis that visual load during SD affects subsequent recovery sleep in human subjects. ${ }^{170}$ Following the condition of high visual load, a significant increase in stage 4 sleep during recovery nights was identified. In another study, a group of healthy subjects and insomniacs were subjected to 64-hour SD, and a decline in the performance measures based on reaction time and immediate recall in both groups was found, while subjective sleepiness increased. ${ }^{171}$ Notably, the behavioral variables were restored after 8 hours of sleep, although it took longer for sleepiness to renormalize. Recently, SWS disruption was found to primarily lead to an increase in sleepiness with only minor effects on other aspects of daytime functioning. ${ }^{172}$ PVT measures and sleepiness have been shown to recover at different rates following sleep restriction, ${ }^{173}$ where the amount of sleep during the recovery period was an essential variable. ${ }^{174}$ Somewhat different results have been obtained in a study, where after $\mathrm{SD}$, subjective sleepiness nearly recovered to the baseline level on the first recovery day, but fatigue and performance in a high-order cognitive task took 2 days to return to baseline levels. ${ }^{175}$ This was supported by another study which reported that the negative effects of SD on task-switching performance were reversed by only one night of recovery sleep. ${ }^{176}$ Similarly, in another protocol where the durations of sleep and wake episodes were increased to 10 and 32.85 hours, respectively, to yield a reduced sleep-to-wake ratio of $1: 3.3$, sleep consistently restored vigilance task performance during the first several hours of wakefulness. ${ }^{177}$

Interestingly, there is evidence that even short periods of sleep can be beneficial. Specifically, a short afternoon nap ( $<30$ minutes) significantly reduced subjective sleepiness and fatigue in the afternoon both in elderly and in young subjects, while subjective mood and performance in a task were also improved. ${ }^{178,179}$ Nevertheless, it remains unclear whether the improvements or restoration observed after sleep reflects an active recovery process obtained during sleep, or a diminished drive to initiate sleep.

\section{What makes sleep after SD a "recovery sleep"?}

Sleep after SD is usually enhanced, with respect to both its total duration and its intensity as measured with EEG SWA, ${ }^{96,141,180,181}$ although notable differences have been found between studies, species, genetic backgrounds, age, the time of day, environmental conditions, and the method used to perform SD. ${ }^{97,182-192}$ Moreover, substantial interindividual variability in the response to $\mathrm{SD}$ has also been found. ${ }^{35,66}$

The terminology used to denote the response to SD would benefit from clarification and improvement. On the one hand, it can be argued that if sleep is necessary to provide recovery, 
eg, if it compensates for preceding waking, then all sleep is "recovery sleep", irrespective of the duration of previous waking. On the other hand, it cannot be excluded that deep intense sleep after SD may have higher "recovery" value, and recovery will be delayed under circumstances where this recovery is prevented, such as in selective slow-wave deprivation experiments, ${ }^{193}$ or when SWS is eliminated specifically during the first part of the night. ${ }^{194}$ Local use-dependent changes in SWA after selective peripheral stimulation have also been considered as evidence for a higher need of local "recovery", ${ }^{64}$ although no causal experiments preventing local increases of SWA after peripheral stimulation have been performed to date.

There have been several attempts to manipulate SD protocols to investigate the subsequent recovery sleep. In one study, physostigmine was administered during SD to induce REM-like sleep in awake rats, while atropine was used to produce EEG slow waves typical of those found during NREM sleep. ${ }^{195}$ These manipulations did not substantially affect subsequent recovery sleep, and it was concluded that pharmacologically induced sleep-like patterns cannot substitute for physiological sleep. However, in another study, sleep characteristics after 6 hours of propofol anesthesia following SD were similar to undisturbed recovery sleep. ${ }^{196}$ A more recent study found that recovery sleep after isoflurane or desflurane anesthesia administered for 1 hour at the end of SD was similar to spontaneous sleep, suggesting that merely the presence of slow waves, or reduced neuronal activity may be sufficient to achieve at least initial recovery. ${ }^{197}$ The active role of slow waves in providing synaptic renormalization was suggested by the finding of a correlation between sleep intensity and changes in cortical evoked potentials across sleep. ${ }^{198}$ Typically, slow waves are absent during active waking, although in some cases a dissociation between behavior and cortical activity can be induced pharmacologically, such as by administration of muscimol or atropine. ${ }^{199,200}$ The role of pharmacologically induced slow waves in subsequent sleep is not entirely clear, mainly because it is often difficult to dissociate residual drug effects from the effects of altered preceding state. However, pharmacological "induction" of wakefulness led to an expected increase in sleep time and EEG SWA during subsequent sleep, which suggests that the dynamics of the underlying regulatory processes were not altered. ${ }^{201,202}$

These findings are consistent with a relationship between brain activity during SD and subsequent sleep, although it should be noted that this relationship is often not straightforward. For example, an increased leakage of slow waves into waking EEG may discharge sleep pressure to some extent, or slow down the buildup of sleep pressure. ${ }^{203}$ The presence of "sleep-like" activity in an otherwise awake brain may however also merely be a reflection of a "sleepdeprived" state and indicate a tendency to initiate sleep, ${ }^{183,204}$ while by itself contributing little to the dissipation of sleep need. Recently, it has been proposed that the occurrence of sleep-like activities during waking is a manifestation of the process of prophylactic cellular maintenance. ${ }^{5}$ According to this hypothesis, and consistent with previous models, ${ }^{205}$ individual neurons and local networks tend to transition into a sleep-like state after a specific amount of activity, which may serve to re-allocate energy substrates essential for cellular maintenance processes. 5,206 The occurrence of such sleep-like events would be expected to affect the entire network dynamics, and could precipitate other deficits. Alternatively, this activity may in theory permit extension of the time spent awake by performing "online" maintenance.

While there is considerable evidence that "recovery" sleep occurs as a result of or is related to preceding waking activities, some notable exceptions under both normal and pathological conditions are well documented. For example, individuals in a persistent vegetative state with greatly impoverished waking that may require little subsequent recovery sleep have been found to have partially preserved sleep-wake cycles. ${ }^{207,208}$ Evidence of increased SWA during the initial sleep after hibernation or torpor suggests that the mere absence of SWA and physiological sleep may be sufficient to necessitate recovery processes. ${ }^{209,210}$ Finally, it cannot be excluded that at least some of sleep's functions may be performed in other states. For example, a recent study found that a period of meditation does not only temporarily restore behavioral performance, but may also lead to a reduced sleep time, suggesting that it may partially substitute for sleep. ${ }^{211}$

\section{Sleep and metaregulation of physiological homeostasis}

In summary, while the notion that sleep proper is a regulated process remains an important concept, the existence of many factors that profoundly influence sleep, or more precisely, manifest themselves in specific sleep parameters, makes it hard to determine what exactly is being regulated. Likewise, the role of sleep in recovery remains one of the predominant hypotheses, despite existing evidence being insufficient and the likelihood that it will remain so due to several reasons. 
Firstly, sleep is a complex process, ${ }^{4,28}$ making it difficult to dissect the contribution of specific phenomena or temporal and spatial aspects associated with sleep, to its recovery functions with respect to specific physiological variables. Secondly, any attempt to investigate the recovery role of sleep with respect to a specific variable must also take into account other essential factors, such as circadian phase, which are often difficult to disentangle. Further undoubtedly necessary efforts will likely bring important advances in our understanding of molecular and cellular processes that benefit from sleep. It is however essential to keep in mind that sleep (as it is traditionally defined) is ultimately a global behavioral phenomenon and that the beneficial effects of sleep are likely to be found at a higher-order level, rather than in specific independent variables.

An important consideration is that the characteristics currently employed to define sleep are mechanistically directly linked to cerebral activity, despite current debate as to whether sleep benefits and SD harms the body or the brain or both. It should be noted that in laboratory experiments SD is achieved by enforcing exploratory behavior or locomotion, using pharmacological means targeting wakepromoting neuromodulators, or by sensory or direct brain stimulation, ${ }^{187,189,201,202,212-214}$ thus targeting the brain in the first instance. Moreover, the success of SD is determined by monitoring EEG/EMG traces, body posture, and movement or by testing the responsiveness of the subject, ie, by readouts of cerebral activity, rather than by measuring peripheral functions. It is well known, however, that sleep is a major modulator of hormonal levels, metabolic status, and the immune system, ${ }^{215-218}$ while SD or sleep restriction are associated with a wide range of peripheral changes that may be visible, for example, at the level of the transcriptome or metabolome. ${ }^{40,219,220}$ However, unlike the neurophysiological effects of sleep and SD, the changes in the neuroendocrine, metabolic, or immune systems are less easily accessible to direct observation in real time, and therefore are not targeted specifically during SD procedures.

It has been proposed that the temporal organization of sleep and circadian rhythms enable the coordination of a wide range of cellular and metabolic processes, which together enable stability and survival..$^{27,41,221}$ The question, however, remains whether and in fact how different processes interact to achieve homeostasis of specific variables at the cellular level to enable optimal adaptive wakefulness at the level of the whole organism. One possibility is that the regulation of specific physiological variables occurs relatively independently, but since the substrate (the organism) is shared purely addi- tive interactions are observed. An alternative scenario is that rather than sharing the substrate, the key molecules or brain circuits are instead shared between specific functions, and a change in one of them would be accompanied by changes in another. Another possibility, which has not been considered previously, is that an additional level of regulation exists, which could take a form of a metaregulation. In economics, meta-regulation refers to a mode of oversight, where the regulatory agency induces self-regulatory arrangements at the lower levels, without interfering with the rules employed downstream. An essential aspect in this form of regulation is the ultimate goal that has to be set, which could be the maintenance of physiological homeostasis in general or adaptive wakefulness in particular. According to this view, sleep is not a distinct entity, which is regulated independently and has a specific function, but, at any given moment, represents the process of metaregulation, which reflects an interaction between internal and external factors, preceding history and current homeostatic needs.

This view describes well the role of sleep in enabling adaptive wakefulness without invoking its recovery (or any specific) function and has several theoretical implications. First and foremost, it accounts for sleep being a highly complex hierarchical process, consisting of at least several distinct sub-states, many processes, anatomical structures, and biochemical pathways involved, which share substrates, integrate environmental influences and preceding history, and interact in many ways. Secondly, if adaptive wakefulness, defined in terms of behavior, the probability of survival, and energy homeostasis is the ultimate target of the metaregulation process, then it would be expected that there are multiple ways to achieve it, which can account for inter-species variability and flexibility in characteristics of the states we call waking and sleep. Finally, the changes observed when sleep is insufficient or absent, such as metabolic disturbances or behavioral deficits, could be viewed not as negative consequences of sleep loss, but rather as manifestations of the breakdown of the process of metaregulation, which no longer provides adaptive wakefulness.

\section{Acknowledgments}

This study was supported by Wellcome Trust Strategic Award (098461/Z/12/Z). The author would like to thank Prof AA Borbély for stimulating discussions and critical comments on the manuscript, and Ms LE McKillop for valuable suggestions and for editing the final version of the manuscript. 


\section{Disclosure}

The author reports no conflicts of interest in this work.

\section{References}

1. Brown RE, Basheer R, McKenna JT, Strecker RE, McCarley RW. Control of sleep and wakefulness. Physiol Rev. 2012;92:1087-1187.

2. Siegel JM. Clues to the functions of mammalian sleep. Nature. 2005;437:1264-1271.

3. Cirelli C, Tononi G. Is sleep essential? PLoS Biol. 2008;6:e216.

4. Vassalli A, Dijk DJ. Sleep function: current questions and new approaches. Eur J Neurosci. 2009;29:1830-1841.

5. Vyazovskiy VV, Harris KD. Sleep and the single neuron: the role of global slow oscillations in individual cell rest. Nat Rev Neurosci. 2013;14:443-451.

6. Abel T, Havekes R, Saletin JM, Walker MP. Sleep, plasticity and memory from molecules to whole-brain networks. Curr Biol. 2013;23:R774-R788.

7. Rasch B, Born J. About sleep's role in memory. Physiol Rev. 2013;93: 681-766.

8. Tononi G, Cirelli C. Sleep and the price of plasticity: from synaptic and cellular homeostasis to memory consolidation and integration. Neuron. 2014;81:12-34.

9. Baran B, Pace-Schott EF, Ericson C, Spencer RM. Processing of emotional reactivity and emotional memory over sleep. $J$ Neurosci. 2012;32:1035-1042.

10. Gujar N, McDonald SA, Nishida M, Walker MP. A role for REM sleep in recalibrating the sensitivity of the human brain to specific emotions. Cereb Cortex. 2011;21:115-123.

11. Perogamvros L, Dang-Vu TT, Desseilles M, Schwartz S. Sleep and dreaming are for important matters. Front Psychol. 2013;4:474.

12. Benington JH, Heller HC. Restoration of brain energy metabolism as the function of sleep. Prog Neurobiol. 1995;45:347-360.

13. Berger RJ, Phillips NH. Energy conservation and sleep. Behav Brain Res. 1995;69:65-73.

14. Horne J. REM sleep, energy balance and 'optimal foraging'. Neurosci Biobehav Rev. 2009;33:466-474.

15. Maquet P. Sleep function(s) and cerebral metabolism. Behav Brain Res. 1995;69:75-83

16. Scharf MT, Naidoo N, Zimmerman JE, Pack AI. The energy hypothesis of sleep revisited. Prog Neurobiol. 2008;86:264-280.

17. Schmidt MH. The energy allocation function of sleep: a unifying theory of sleep, torpor, and continuous wakefulness. Neurosci Biobehav Rev. 2014;47:122-153.

18. Mackiewicz M, Shockley KR, Romer MA, et al. Macromolecule biosynthesis: a key function of sleep. Physiol Genomics. 2007;31: 441-457.

19. Varshavsky A. Augmented generation of protein fragments during wakefulness as the molecular cause of sleep: a hypothesis. Protein Sci. 2012;21:1634-1661.

20. Xie L, Kang H, Xu Q, et al. Sleep drives metabolite clearance from the adult brain. Science. 2013;342:373-377.

21. Corner MA. From neural plate to cortical arousal-a neuronal network theory of sleep derived from in vitro "model" systems for primordial patterns of spontaneous bioelectric activity in the vertebrate central nervous system. Brain Sci. 2013;3:800-820.

22. Hinard V, Mikhail C, Pradervand S, et al. Key electrophysiological, molecular, and metabolic signatures of sleep and wakefulness revealed in primary cortical cultures. $J$ Neurosci. 2012;32:12506-12517.

23. Horne JA. REM sleep - by default? Neurosci Biobehav Rev. 2000;24: 777-797.

24. Sanchez-Vives MV, Mattia M. Slow wave activity as the default mode of the cerebral cortex. Arch Ital Biol. 2014;152:147-155.

25. Siegel JM. Sleep viewed as a state of adaptive inactivity. Nat Rev Neurosci. 2009;10:747-753.
26. Tobler I. Is sleep fundamentally different between mammalian species? Behav Brain Res. 1995;69:35-41.

27. Mignot E. Why we sleep: the temporal organization of recovery. PLoS Biol. 2008;6:e106.

28. Vyazovskiy VV, Delogu A. NREM and REM sleep: complementary roles in recovery after wakefulness. Neuroscientist. 2014;20:203-219.

29. Borbély AA, Achermann P. Sleep homeostasis and models of sleep regulation. In: Kryger MH, Roth T, Derment WC, editors. Principles and Practice of Sleep Medicine. Philadelphia: WB Saunders; 2005: 405-417.

30. Abraham WC, Bear MF. Metaplasticity: the plasticity of synaptic plasticity. Trends Neurosci. 1996;19:126-130.

31. Steriade M,Amzica F. Coalescence of sleep rhythms and their chronology in corticothalamic networks. Sleep Res Online. 1998;1:1-10.

32. Van Cauter E, Latta F, Nedeltcheva A, et al. Reciprocal interactions between the GH axis and sleep. Growth Horm IGF Res. 2004;14(SupplA): S10-S17.

33. Campbell SS, Tobler I. Animal sleep: a review of sleep duration across phylogeny. Neurosci Biobehav Rev. 1984;8:269-300.

34. Juda M, Vetter C, Roenneberg T. Chronotype modulates sleep duration, sleep quality, and social jet lag in shift-workers. J Biol Rhythms. 2013;28:141-151.

35. Rusterholz T, Durr R, Achermann P. Inter-individual differences in the dynamics of sleep homeostasis. Sleep. 2010;33:491-498.

36. Van Dongen HP, Vitellaro KM, Dinges DF. Individual differences in adult human sleep and wakefulness: Leitmotif for a research agenda. Sleep. 2005;28:479-496.

37. Vyazovskiy VV, Tobler I. The temporal structure of behaviour and sleep homeostasis. PLoS One. 2012;7:e50677.

38. Franken P. A role for clock genes in sleep homeostasis. Curr Opin Neurobiol. 2013;23:864-872.

39. Mongrain V, La Spada F, Curie T, Franken P. Sleep loss reduces the DNA-binding of BMAL1, CLOCK, and NPAS2 to specific clock genes in the mouse cerebral cortex. PLoS One. 2011;6:e26622.

40. Archer SN, Laing EE, Moller-Levet CS, et al. Mistimed sleep disrupts circadian regulation of the human transcriptome. Proc Natl Acad Sci U S A. 2014;111:E682-E691.

41. Bass J, Takahashi JS. Circadian integration of metabolism and energetics. Science. 2010;330:1349-1354.

42. Adamantidis A, de Lecea L. Sleep and metabolism: shared circuits, new connections. Trends Endocrinol Metab. 2008;19:362-370.

43. Burdakov D, Karnani MM, Gonzalez A. Lateral hypothalamus as a sensor-regulator in respiratory and metabolic control. Physiol Behav. 2013;121:117-124.

44. Kosse C, Burdakov D. A unifying computational framework for stability and flexibility of arousal. Front Syst Neurosci. 2014;8:192.

45. Saper CB. Staying awake for dinner: hypothalamic integration of sleep, feeding, and circadian rhythms. Prog Brain Res. 2006;153:243-252.

46. Greenspan RJ, Tononi G, Cirelli C, Shaw PJ. Sleep and the fruit fly. Trends Neurosci. 2001;24:142-145.

47. Polack PO, Friedman J, Golshani P. Cellular mechanisms of brain state-dependent gain modulation in visual cortex. Nat Neurosci. 2013;16:1331-1339.

48. Poulet JF, Petersen CC. Internal brain state regulates membrane potential synchrony in barrel cortex of behaving mice. Nature. 2008;454:881-885.

49. Zagha E, Casale AE, Sachdev RN, McGinley MJ, McCormick DA. Motor cortex feedback influences sensory processing by modulating network state. Neuron. 2013;79:567-578.

50. Grush R. The emulation theory of representation: motor control, imagery, and perception. Behav Brain Sci. 2004;27:377-396; discussion 396-442.

51. Churchland PS. Brain-Wise: Studies in Neurophilosophy. Cambridge, MA: MIT Press; 2002.

52. Pigarev IN, Nothdurft HC, Kastner S. Evidence for asynchronous development of sleep in cortical areas. Neuroreport. 1997;8:2557-2560. 
53. NirY, Vyazovskiy VV, Cirelli C, Banks MI, Tononi G. Auditory responses and stimulus-specific adaptation in rat auditory cortex are preserved across NREM and REM sleep. Cereb Cortex. 2015;25:1362-1378.

54. Vyazovskiy VV, Faraguna U, Cirelli C, Tononi G. Triggering slow waves during NREM sleep in the rat by intracortical electrical stimulation: effects of sleep/wake history and background activity. J Neurophysiol. 2009;101:1921-1931.

55. Saper CB, Fuller PM, Pedersen NP, Lu J, Scammell TE. Sleep state switching. Neuron. 2010;68:1023-1042.

56. Mileykovskiy BY, Kiyashchenko LI, Siegel JM. Behavioral correlates of activity in identified hypocretin/orexin neurons. Neuron. 2005;46: 787-798.

57. Takahashi K, Kayama Y, Lin JS, Sakai K. Locus coeruleus neuronal activity during the sleep-waking cycle in mice. Neuroscience. 2010;169: 1115-1126.

58. Takahashi K, Lin JS, Sakai K. Neuronal activity of orexin and nonorexin waking-active neurons during wake-sleep states in the mouse. Neuroscience. 2008;153:860-870.

59. Carter ME, Adamantidis A, Ohtsu H, Deisseroth K, de Lecea L. Sleep homeostasis modulates hypocretin-mediated sleep-to-wake transitions. J Neurosci. 2009;29:10939-10949.

60. Kouider S, Andrillon T, Barbosa LS, Goupil L, Bekinschtein TA. Inducing task-relevant responses to speech in the sleeping brain. Curr Biol. 2014;24:2208-2214.

61. Bellesi M, Tononi G, Cirelli C, Serra PA. Region-specific dissociation between cortical noradrenaline levels and the sleep/wake cycle. Sleep. Epub 2015 Jul 24.

62. de Lecea L, Carter ME, Adamantidis A. Shining light on wakefulness and arousal. Biol Psychiatry. 2012;71:1046-1052.

63. Lee SH, Dan Y. Neuromodulation of brain states. Neuron. 2012;76:209-222.

64. McCormick DA. Neurotransmitter actions in the thalamus and cerebral cortex and their role in neuromodulation of thalamocortical activity. Prog Neurobiol. 1992;39:337-388.

65. Killgore WD. Effects of sleep deprivation on cognition. Prog Brain Res. 2010;185:105-129.

66. Lo JC, Groeger JA, Santhi N, et al. Effects of partial and acute total sleep deprivation on performance across cognitive domains, individuals and circadian phase. PLoS One. 2012;7:e45987.

67. Van Dongen HP, Belenky G, Krueger JM. A local, bottom-up perspective on sleep deprivation and neurobehavioral performance. Curr Top Med Chem. 2011;11:2414-2422.

68. Jones BE. From waking to sleeping: neuronal and chemical substrates Trends Pharmacol Sci. 2005;26:578-586.

69. Nir Y, Tononi G. Dreaming and the brain: from phenomenology to neurophysiology. Trends Cogn Sci. 2010;14:88-100.

70. Davis CJ, Clinton JM, Jewett, KA, Zielinski MR, Krueger JM. Delta wave power: an independent sleep phenotype or epiphenomenon? J Clin Sleep Med. 2011;7:S16-S18.

71. Greene RW, Frank MG. Slow wave activity during sleep: functional and therapeutic implications. Neuroscientist. 2010;16:618-633.

72. Sanchez-Vives MV, McCormick DA. Cellular and network mechanisms of rhythmic recurrent activity in neocortex. Nat Neurosci. 2000;3: 1027-1034.

73. Contreras D, Steriade M. Cellular basis of EEG slow rhythms: a study of dynamic corticothalamic relationships. J Neurosci. 1995;15: 604-622.

74. Murphy M, Bruno MA, Riedner BA, et al. Propofol anesthesia and sleep: a high-density EEG study. Sleep. 2011;34:283A-291A.

75. Timofeev I, Grenier F, Bazhenov M, Sejnowski TJ, Steriade M. Origin of slow cortical oscillations in deafferented cortical slabs. Cereb Cortex. 2000;10:1185-1199.

76. Constantinople CM, Bruno RM. Effects and mechanisms of wakefulness on local cortical networks. Neuron. 2011;69:1061-1068.

77. Bellesi M, Riedner BA, Garcia-Molina GN, Cirelli C, Tononi G. Enhancement of sleep slow waves: underlying mechanisms and practical consequences. Front Syst Neurosci. 2014;8:208.
78. Massimini M, Ferrarelli F, Esser SK, et al. Triggering sleep slow waves by transcranial magnetic stimulation. Proc Natl Acad Sci US A. 2007;104:8496-8501.

79. Riedner BA, Hulse BK, Murphy MJ, Ferrarelli F, Tononi G. Temporal dynamics of cortical sources underlying spontaneous and peripherally evoked slow waves. Prog Brain Res. 2011;193:201-218.

80. Vyazovskiy VV, Olcese U, Cirelli C, Tononi G. Prolonged wakefulness alters neuronal responsiveness to local electrical stimulation of the neocortex in awake rats. $J$ Sleep Res. 2013;22:264-271.

81. Lemieux M, Chen JY, Lonjers P, Bazhenov M, Timofeev I. The impact of cortical deafferentation on the neocortical slow oscillation. J Neurosci. 2014;34:5689-5703.

82. Zhang Z, Ferretti V, Guntan I, et al. Neuronal ensembles sufficient for recovery sleep and the sedative actions of alpha2 adrenergic agonists. Nat Neurosci. 2015;18:553-561.

83. Mahowald MW, Cramer Bornemann MA, Schenck CH. State dissociation, human behavior, and consciousness. Curr Top Med Chem. 2011;11:2392-2402.

84. Vanderwolf $\mathrm{CH}$. The electrocorticogram in relation to physiology and behavior: a new analysis. Electroencephalogr Clin Neurophysiol. 1992;82:165-175.

85. Cui N, McKillop LE, Fisher SP, Oliver PL, Vyazovskiy VV. Longterm history and immediate preceding state affect EEG slow wave characteristics at NREM sleep onset in C57BL/6 mice. Arch Ital Biol. 2014;152:156-168.

86. Vyazovskiy VV, Cui N, Rodriguez AV, Funk C, Cirelli C, Tononi G. The dynamics of cortical neuronal activity in the first minutes after spontaneous awakening in rats and mice. Sleep. 2014;37: 1337-1347.

87. Achermann P, Dijk DJ, Brunner DP, Borbely AA. A model of human sleep homeostasis based on EEG slow-wave activity: quantitative comparison of data and simulations. Brain Res Bull. 1993;31: 97-113.

88. Borbély AA. A two process model of sleep regulation. Hum Neurobiol. 1982;1:195-204.

89. Daan S, Beersma DG, Borbely AA. Timing of human sleep: recovery process gated by a circadian pacemaker. Am J Physiol. 1984;246: R161-R183.

90. Steriade M. Cholinergic blockage of network- and intrinsically generated slow oscillations promotes waking and REM sleep activity patterns in thalamic and cortical neurons. Prog Brain Res. 1993;98: $345-355$.

91. Deboer T. Behavioral and electrophysiological correlates of sleep and sleep homeostasis. Curr Top Behav Neurosci. 2015;25:1-24.

92. Webb WB, Agnew HW Jr. Sleep: effects of a restricted regime. Science. 1965;150:1745-1747.

93. Nakazawa Y, Kotorii M, Ohshima M, Kotorii T, Hasuzawa H. Changes in sleep pattern after sleep deprivation. Folia Psychiatr Neurol Jpn. 1978;32:85-93.

94. Takahashi Y, Ebihara S, Nakamura Y, Takahashi K. Temporal distributions of delta wave sleep and rem sleep during recovery sleep after 12-h forced wakefulness in dogs; similarity to human sleep. Neurosci Lett. 1978;10:329-334.

95. Borbely AA, Baumann F, Brandeis D, Strauch I, Lehmann D. Sleep deprivation: effect on sleep stages and EEG power density in man. Electroencephalogr Clin Neurophysiol. 1981;51:483-495.

96. Tobler I, Borbely AA. Sleep EEG in the rat as a function of prior waking. Electroencephalogr Clin Neurophysiol. 1986;64:74-76.

97. Huber R, Deboer T, Tobler I. Effects of sleep deprivation on sleep and sleep EEG in three mouse strains: empirical data and simulations. Brain Res. 2000;857:8-19.

98. Vyazovskiy VV, Ruijgrok G, Deboer T, Tobler I. Running wheel accessibility affects the regional electroencephalogram during sleep in mice. Cereb Cortex. 2006;16:328-336.

99. Vyazovskiy VV, Achermann P, Tobler I. Sleep homeostasis in the rat in the light and dark period. Brain Res Bull. 2007;74:37-44. 
100. Achermann P, Borbely AA. Low-frequency $(<1 \mathrm{~Hz})$ oscillations in the human sleep electroencephalogram. Neuroscience. 1997;81: 213-222.

101. Achermann P, Borbely AA. Temporal evolution of coherence and power in the human sleep electroencephalogram. J Sleep Res. 1998;7(Suppl 1):36-41.

102. Campbell IG, Higgins LM, Darchia N, Feinberg I. Homeostatic behavior of fast Fourier transform power in very low frequency nonrapid eye movement human electroencephalogram. Neuroscience. 2006;140:1395-1399.

103. Dijk DJ, Beersma DG, Daan S. EEG power density during nap sleep: reflection of an hourglass measuring the duration of prior wakefulness. J Biol Rhythms. 1987;2:207-219.

104. Vyazovskiy VV, Riedner BA, Cirelli C, Tononi G. Sleep homeostasis and cortical synchronization: II. A local field potential study of sleep slow waves in the rat. Sleep. 2007;30:1631-1642.

105. Bergmann BM, Mistlberger RE, Rechtschaffen A. Period-amplitude analysis of rat electroencephalogram: stage and diurnal variations and effects of suprachiasmatic nuclei lesions. Sleep. 1987;10:523-536.

106. Feinberg I, March JD, Fein G, Floyd TC, Walker JM, Price L. Period and amplitude analysis of $0.5-3 \mathrm{c} / \mathrm{sec}$ activity in NREM sleep of young adults. Electroencephalogr Clin Neurophysiol. 1978;44: 202-213.

107. Mistlberger R, Bergmann B, Rechtschaffen A. Period-amplitude analysis of rat electroencephalogram: effects of sleep deprivation and exercise. Sleep. 1987;10:508-522.

108. Bersagliere A, Achermann P. Slow oscillations in human non-rapid eye movement sleep electroencephalogram: effects of increased sleep pressure. J Sleep Res. 2010;19(1 Pt 2):228-237.

109. Riedner BA, Vyazovskiy VV, Huber R, et al. Sleep homeostasis and cortical synchronization: III. A high-density EEG study of sleep slow waves in humans. Sleep. 2007;30:1643-1657.

110. Vyazovskiy VV, Cirelli C, Tononi G. Electrophysiological correlates of sleep homeostasis in freely behaving rats. Prog Brain Res. 2011;193: $17-38$.

111. Vyazovskiy VV, Olcese U, Lazimy YM, et al. Cortical firing and sleep homeostasis. Neuron. 2009;63:865-878.

112. Tobler I. Phylogeny of sleep regulation. In: Kryger MH, Roth T, Derment WC, editors. Principles and Practice of Sleep Medicine. Philadelphia: WB Saunders; 2005.

113. Petit JM, Tobler I, Allaman I, Borbely AA, Magistretti PJ. Sleep deprivation modulates brain mRNAs encoding genes of glycogen metabolism. Eur J Neurosci. 2002;16:1163-1167.

114. Porkka-Heiskanen T, Strecker RE, Thakkar M, Bjorkum AA, Greene RW, McCarley RW. Adenosine: a mediator of the sleep-inducing effects of prolonged wakefulness. Science. 1997;276:1265-1268.

115. Ramanathan L, Siegel JM. Sleep deprivation under sustained hypoxia protects against oxidative stress. Free Radic Biol Med. 2011;51: 1842-1848.

116. Clinton JM, Davis CJ, Zielinski MR, Jewett KA, Krueger JM. Biochemical regulation of sleep and sleep biomarkers. J Clin Sleep Med. 2011;7:S38-S42.

117. Obal F Jr, Krueger JM. Biochemical regulation of non-rapid-eyemovement sleep. Front Biosci. 2003;8:d520-d550.

118. Krueger JM, Rector DM, Roy S, Van Dongen HP, Belenky G, Panksepp J. Sleep as a fundamental property of neuronal assemblies. Nat Rev Neurosci. 2008;9:910-919.

119. Tononi G, Cirelli C. Sleep function and synaptic homeostasis. Sleep Med Rev. 1993;10:49-62.

120. Cirelli C, Gutierrez CM, Tononi G. Extensive and divergent effects of sleep and wakefulness on brain gene expression. Neuron. 2004;41:35-43.

121. Cirelli C, Tononi G. Gene expression in the brain across the sleepwaking cycle. Brain Res. 2000;885:303-321.

122. Mackiewicz M, Naidoo N, Zimmerman JE, Pack AI. Molecular mechanisms of sleep and wakefulness. Ann NY Acad Sci. 2008;1129: $335-349$.
123. Cajochen C, Wyatt JK, Czeisler CA, Dijk DJ. Separation of circadian and wake duration-dependent modulation of EEG activation during wakefulness. Neuroscience. 2002;114:1047-1060.

124. Vyazovskiy VV, Tobler I. Handedness leads to interhemispheric EEG asymmetry during sleep in the rat. J Neurophysiol. 2008;99: 969-975.

125. Dijk DJ, Czeisler CA. Contribution of the circadian pacemaker and the sleep homeostat to sleep propensity, sleep structure, electroencephalographic slow waves, and sleep spindle activity in humans. J Neurosci. 1995;15:3526-3538.

126. Lazar AS, Lazar ZI, Dijk DJ. Circadian regulation of slow waves in human sleep: topographical aspects. Neuroimage. 2015;116: 123-134.

127. Tobler I, Franken P, Alfoldi P, Borbely AA. Room light impairs sleep in the albino rat. Behav Brain Res. 1994;63:205-211.

128. Gao BO, Franken P, Tobler I, Borbely AA. Effect of elevated ambient temperature on sleep, EEG spectra, and brain temperature in the rat. Am J Physiol. 1995;268:R1365-R1373.

129. Groeger JA, Lo JC, Burns CG, Dijk DJ. Effects of sleep inertia after daytime naps vary with executive load and time of day. Behav Neurosci. 2011;125:252-260.

130. Chauvette S, Seigneur J, Timofeev I. Sleep oscillations in the thalamocortical system induce long-term neuronal plasticity. Neuron. 2012;75: 1105-1113.

131. Durkin J, Aton SJ. Sleep-dependent potentiation in the visual system is at odds with the synaptic homeostasis hypothesis. Sleep. Epub 2015 Aug 12.

132. Frank MG. Erasing synapses in sleep: is it time to be SHY? Neural Plast. 2012;2012:264378.

133. Tononi G, Cirelli C. Time to be SHY? Some comments on sleep and synaptic homeostasis. Neural Plast. 2012;2012:415250.

134. Horne J. Exercise benefits for the aging brain depend on the accompanying cognitive load: insights from sleep electroencephalogram. Sleep Med. 2013;14:1208-1213.

135. Hobson JA. Sleep after exercise. Science. 1968;162:1503-1505.

136. Levitt RA. Sleep deprivation in the rat. Science. 1966;153:85-87.

137. Shapiro CM, Griesel RD, Bartel PR, Jooste PL. Sleep patterns afted graded exercise. J Appl Physiol. 1975;39:187-190.

138. Shapiro CM, Bortz R, Mitchell D, Bartel P, Jooste P. Slow-wave sleep: a recovery period after exercise. Science. 1981;214:1253-1254.

139. Torsvall L, Akerstedt T, Lindbeck G. Effects on sleep stages and EEG power density of different degrees of exercise in fit subjects. Electroencephalogr Clin Neurophysiol. 1984;57:347-353.

140. Horne JA, Porter JM. Time of day effects with standardized exercise upon subsequent sleep. Electroencephalogr Clin Neurophysiol. 1976;40:178-184.

141. Friedman L, Bergmann BM, Rechtschaffen A. Effects of sleep deprivation on sleepiness, sleep intensity, and subsequent sleep in the rat. Sleep. 1979;1:369-391.

142. Borbely AA, Neuhaus HU. Sleep deprivation: effects on sleep and EEG in the rat. J Comp Physiol. 1979;133:71-87.

143. Hanagasioglu M, Borbely AA. Effect of voluntary locomotor activity on sleep in the rat. Behav Brain Res. 1982;4:359-368.

144. Susic V, Kovacevic-Ristanovic R. Effects of restricted sleep with different exercise loads upon subsequent sleep. Arch Int Physiol Biochim. 1980;88:1-13.

145. Buguet A, Roussel B, Angus R, Sabiston B, Radomski M. Human sleep and adrenal individual reactions to exercise. Electroencephalogr Clin Neurophysiol. 1980;49:515-523.

146. Youngstedt SD, Kripke DF, Elliott JA. Is sleep disturbed by vigorous late-night exercise? Med Sci Sports Exerc. 1999;31:864-869.

147. Ritsche K, Nindl BC, Wideman L. Exercise-Induced growth hormone during acute sleep deprivation. Physiol Rep. Epub 2014 Oct 2.

148. McFadden KL, Cornier MA, Melanson EL, Bechtell JL, Tregellas JR. Effects of exercise on resting-state default mode and salience network activity in overweight/obese adults. Neuroreport. 2013;24: 866-871. 
149. Voss MW, Prakash RS, Erickson KI, et al. Plasticity of brain networks in a randomized intervention trial of exercise training in older adults. Front Aging Neurosci. Epub 2010 Aug 26.

150. Vyazovskiy VV. Cortical neuronal mechanisms of sleep homeostasis. Zh Vyssh Nerv Deiat Im I P Pavlova. 2013;63:13-23.

151. Edgar DM, Kilduff TS, Martin CE, Dement WC. Influence of running wheel activity on free-running sleep/wake and drinking circadian rhythms in mice. Physiol Behav. 1991;50:373-378.

152. Gu C, Coomans CP, Hu K, Scheer FA, Stanley HE, Meijer JH. Lack of exercise leads to significant and reversible loss of scale invariance in both aged and young mice. Proc Natl Acad Sci U S A. 2015;112:2320-2324.

153. Goel N, Rao H, Durmer JS, Dinges DF. Neurocognitive consequences of sleep deprivation. Semin Neurol. 2009;29:320-339.

154. Van Dongen HP, Maislin G, Mullington JM, Dinges DF. The cumulative cost of additional wakefulness: dose-response effects on neurobehavioral functions and sleep physiology from chronic sleep restriction and total sleep deprivation. Sleep. 2003;26:117-126.

155. Fisher SP, Vyazovskiy VV. Local sleep taking care of high-maintenance cortical circuits under sleep restriction. Sleep. 2014;37: 1727-1730.

156. Halasz P, Bodizs R, Parrino L, Terzano M. Two features of sleep slow waves: homeostatic and reactive aspects - from long term to instant sleep homeostasis. Sleep Med. 2014;15:1184-1195.

157. Cajochen C, Foy R, Dijk DJ. Frontal predominance of a relative increase in sleep delta and theta EEG activity after sleep loss in humans. Sleep Res Online. 1999;2:65-69.

158. Finelli LA, Borbely AA, Achermann P. Functional topography of the human nonREM sleep electroencephalogram. Eur J Neurosci. 2001;13:2282-2290.

159. Huber R, Deboer T, Tobler I. Topography of EEG dynamics after sleep deprivation in mice. J Neurophysiol. 2000;84:1888-1893.

160. Vyazovskiy VV, Borbely AA, Tobler I. Interhemispheric sleep EEG asymmetry in the rat is enhanced by sleep deprivation. J Neurophysiol. 2002;88:2280-2286.

161. Werth E, Achermann P, Borbely AA. Brain topography of the human sleep EEG: antero-posterior shifts of spectral power. Neuroreport. 1996;8:123-127.

162. Zavada A, Strijkstra AM, Boerema AS, Daan S, Beersma DG. Evidence for differential human slow-wave activity regulation across the brain. J Sleep Res. 2009;18:3-10.

163. Kattler H, Dijk DJ, Borbely AA. Effect of unilateral somatosensory stimulation prior to sleep on the sleep EEG in humans. J Sleep Res. 1994;3:159-164.

164. Vyazovskiy V, Borbely AA, Tobler I. Unilateral vibrissae stimulation during waking induces interhemispheric EEG asymmetry during subsequent sleep in the rat. J Sleep Res. 2000;9:367-371.

165. Vyazovskiy VV, Welker E, Fritschy JM, Tobler I. Regional pattern of metabolic activation is reflected in the sleep EEG after sleep deprivation combined with unilateral whisker stimulation in mice. Eur $J$ Neurosci. 2004;20:1363-1370.

166. Hanlon EC, Faraguna U, Vyazovskiy VV, Tononi G, Cirelli C. Effects of skilled training on sleep slow wave activity and cortical gene expression in the rat. Sleep. 2009;32:719-729.

167. Huber R, Ghilardi MF, Massimini M, Tononi G. Local sleep and learning. Nature. 2004;430:78-81.

168. Huber R, Ghilardi MF, Massimini M, et al. Arm immobilization causes cortical plastic changes and locally decreases sleep slow wave activity. Nat Neurosci. 2006;9:1169-1176.

169. Krueger JM, Huang YH, Rector DM, Buysse DJ. Sleep: a synchrony of cell activity-driven small network states. Eur J Neurosci. 2013;38:2199-2209.

170. Horne JA. Recovery sleep following different visual conditions during total sleep deprivation in man. Biol Psychol. 1976;4:107-118.

171. Bonnet MH. Recovery of performance during sleep following sleep deprivation in older normal and insomniac adult males. Percept Mot Skills. 1985;60:323-334.
172. Groeger JA, Stanley N, Deacon S, Dijk DJ. Dissociating effects of global SWS disruption and healthy aging on waking performance and daytime sleepiness. Sleep. 2014;37:1127-1142.

173. Lamond N, Jay SM, Dorrian J, Ferguson SA, Jones C, Dawson D. The dynamics of neurobehavioural recovery following sleep loss. J Sleep Res. 2007;16:33-41.

174. Jay SM, Lamond N, Ferguson SA, Dorrian J, Jones CB, Dawson D. The characteristics of recovery sleep when recovery opportunity is restricted. Sleep. 2007;30:353-360.

175. Ikegami K, Ogyu S, Arakomo Y, et al. Recovery of cognitive performance and fatigue after one night of sleep deprivation. J Occup Health. 2009;51:412-422.

176. Couyoumdjian A, Sdoia S, Tempesta D. The effects of sleep and sleep deprivation on task-switching performance. J Sleep Res. 2010;19:64-70.

177. Cohen DA, Wang W, Wyatt JK. Uncovering residual effects of chronic sleep loss on human performance. Sci Transl Med. 2010;2: 14 ra13.

178. Hayashi M, Motoyoshi N, Hori T. Recuperative power of a short daytime nap with or without stage 2 sleep. Sleep. 2005;28: 829-836.

179. Tamaki M, Shirota A, Hayashi M, Hori T. Restorative effects of a short afternoon nap ( $<30 \mathrm{~min}$ ) in the elderly on subjective mood, performance and EEG activity. Sleep Res Online. 2000;3: 131-139.

180. Borbely AA, Tobler I, Hanagasioglu M. Effect of sleep deprivation on sleep and EEG power spectra in the rat. Behav Brain Res. 1984;14:171-182.

181. Mistlberger RE, Bergmann BM, Waldenar W, Rechtschaffen A. Recovery sleep following sleep deprivation in intact and suprachiasmatic nuclei-lesioned rats. Sleep. 1983;6:217-233.

182. Alfoldi P, Tobler I, Borbely AA . Sleep regulation in rats during early development. Am J Physiol. 1990;258:R634-R644.

183. Benington $\mathrm{JH}, \mathrm{Heller} \mathrm{HC}$. Implications of sleep deprivation experiments for our understanding of sleep homeostasis. Sleep. 1999;22:1033-1043.

184. Curie T, Mongrain V, Dorsaz S, Mang GM, Emmenegger Y, Franken P. Homeostatic and circadian contribution to EEG and molecular state variables of sleep regulation. Sleep. 2013;36:311-323.

185. Deboer T, Franken P, Tobler I. Sleep and cortical temperature in the Djungarian hamster under baseline conditions and after sleep deprivation. J Comp Physiol A. 1994;174:145-155.

186. Frank MG, Morrissette R, Heller HC. Effects of sleep deprivation in neonatal rats. Am J Physiol. 1998;275:R148-R157.

187. Huber R, Tononi G, Cirelli C. Exploratory behavior, cortical BDNF expression, and sleep homeostasis. Sleep. 2007;30:129-139.

188. Lafortune M, Gagnon JF, Latreille V, et al. Reduced slow-wave rebound during daytime recovery sleep in middle-aged subjects. PLoS One. 2012;7:e43224.

189. Rechtschaffen A, Bergmann BM, Gilliland MA, Bauer K. Effects of method, duration, and sleep stage on rebounds from sleep deprivation in the rat. Sleep. 1999;22:11-31.

190. Tobler I, Borbely AA, Groos G. The effect of sleep deprivation on sleep in rats with suprachiasmatic lesions. Neurosci Lett. 1983;42: 49-54.

191. Tobler I, Franken P, Jaggi K. Vigilance states, EEG spectra, and cortical temperature in the guinea pig. Am J Physiol. 1993;264: R1125-R1132.

192. Trachsel L, Tobler I, Borbely AA. Sleep regulation in rats: effects of sleep deprivation, light, and circadian phase. Am J Physiol. 1986;251: R1037-R1044.

193. Endo T, Schwierin B, Borbely AA, Tobler I. Selective and total sleep deprivation: effect on the sleep EEG in the rat. Psychiatry Res. 1997;66:97-110.

194. Dijk DJ, Beersma DG. Effects of SWS deprivation on subsequent EEG power density and spontaneous sleep duration. Electroencephalogr Clin Neurophysiol. 1989;72:312-320. 
195. Radil-Weiss T. An attempt to influence by drugs recovery sleep after sleep deprivation. Act Nerv Super (Praha). 1975;17:15-19.

196. Tung A, Bergmann BM, Herrera S, Cao D, Mendelson WB. Recovery from sleep deprivation occurs during propofol anesthesia. Anesthesiology. 2004;100:1419-1426.

197. Nelson AB, Faraguna U, Tononi G, Cirelli C. Effects of anesthesia on the response to sleep deprivation. Sleep. 2010;33:1659-1667.

198. Vyazovskiy VV, Cirelli C, Pfister-Genskow M, Faraguna U, Tononi G. Molecular and electrophysiological evidence for net synaptic potentiation in wake and depression in sleep. Nat Neurosci. 2008;11: 200-208.

199. Qiu MH, Chen MC, Lu J. Cortical neuronal activity does not regulate sleep homeostasis. Neuroscience. 2015;297:211-218.

200. Vyazovskiy VV, Tobler I, Winsky-Sommerer R. Alteration of behavior in mice by muscimol is associated with regional electroencephalogram synchronization. Neuroscience. 2007;147:833-841.

201. Kopp C, Petit JM, Magistretti P, Borbely AA, Tobler I. Comparison of the effects of modafinil and sleep deprivation on sleep and cortical EEG spectra in mice. Neuropharmacology. 2002;43:110-118.

202. Schwierin B, Borbely AA, Tobler I. Effects of N6-cyclopentyladenosine and caffeine on sleep regulation in the rat. Eur J Pharmacol. 1996;300:163-171.

203. Leemburg S, Vyazovskiy VV, Olcese U, Bassetti CL, Tononi G, Cirelli C. Sleep homeostasis in the rat is preserved during chronic sleep restriction. Proc Natl Acad Sci U S A. 2010;107:15939-15944.

204. Vyazovskiy VV, Tobler I. Theta activity in the waking EEG is a marker of sleep propensity in the rat. Brain Res. 2005;1050:64-71.

205. Roy S, Krueger JM, Rector DM, Wan Y. A network model for activitydependent sleep regulation. J Theor Biol. 2008;253:462-468.

206. Maret S, Dorsaz S, Gurcel L, et al. Homerla is a core brain molecular correlate of sleep loss. Proc Natl Acad Sci US A. 2007;104:20090-20095.

207. Danze F, Brule JF, Haddad K. Chronic vegetative state after severe head injury: clinical study; electrophysiological investigations and CT scan in 15 cases. Neurosurg Rev. 1989;12(Suppl 1):477-499.

208. Oboler SK. Brain death and persistent vegetative states. Clin Geriatr Med. 1986;2:547-576.
209. Daan S, Barnes BM, Strijkstra AM. Warming up for sleep? Ground squirrels sleep during arousals from hibernation. Neurosci Lett. 1991;128:265-268.

210. Deboer T, Tobler I. Sleep EEG after daily torpor in the Djungarian hamster: similarity to the effect of sleep deprivation. Neurosci Lett. 1994;166:35-38.

211. Kaul P, Passafiume J, Sargent CR, O’Hara BF. Meditation acutely improves psychomotor vigilance, and may decrease sleep need. Behav Brain Funct. 2010;6:47.

212. Benington JH, Woudenberg MC, Heller HC. REM-sleep propensity accumulates during 2-h REM-sleep deprivation in the rest period in rats. Neurosci Lett. 1994;180:76-80.

213. Gross BA, Vanderheyden WM, Urpa LM, et al. Stress-free automatic sleep deprivation using air puffs. J Neurosci Methods. 2015;251: 83-91.

214. Vyazovskiy V, Achermann P, Borbely AA, Tobler I. Interhemispheric coherence of the sleep electroencephalogram in mice with congenital callosal dysgenesis. Neuroscience. 2004;124:481-488.

215. Imeri L, Opp MR. How (and why) the immune system makes us sleep. Nat Rev Neurosci. 2009;10:199-210.

216. Lange T, Dimitrov S, Born J. Effects of sleep and circadian rhythm on the human immune system. Ann N Y Acad Sci. 2010;1193:48-59.

217. Rogers NL, Szuba MP, Staab JP, Evans DL, Dinges DF. Neuroimmunologic aspects of sleep and sleep loss. Semin Clin Neuropsychiatry. 2001;6:295-307.

218. Van Cauter E, Spiegel K, Tasali E, Leproult R. Metabolic consequences of sleep and sleep loss. Sleep Med. 2008;9(Suppl 1):S23-S28.

219. Davies SK, Ang JE, Revell VL, et al. Effect of sleep deprivation on the human metabolome. Proc Natl Acad Sci U S A. 2014;111: 10761-10766.

220. Moller-Levet CS, Archer SN, Bucca G, et al. Effects of insufficient sleep on circadian rhythmicity and expression amplitude of the human blood transcriptome. Proc Natl Acad Sci U S A. 2013;110:E1132-E1141.

221. Frank MG, Cantera R. Sleep, clocks, and synaptic plasticity. Trends Neurosci. 2014;37:491-501.
Nature and Science of Sleep

\section{Publish your work in this journal}

Nature and Science of Sleep is an international, peer-reviewed, open access journal covering all aspects of sleep science and sleep medicine, including the neurophysiology and functions of sleep, the genetics of sleep, sleep and society, biological rhythms, dreaming, sleep disorders and therapy, and strategies to optimize healthy sleep. The journal welcomes

\section{Dovepress}

original research, clinical \& epidemiological studies, reviews \& evaluations, case reports and extended reports. The manuscript management system is completely online and includes a very quick and fair peerreview system, which is all easy to use. Visit http://www.dovepress.com/ testimonials.php to read real quotes from published authors. 